\title{
Tendências em medicina laboratorial
}

\author{
Trends in laboratory medicine
}

Primeira submissão em 07/12/09 Última submissão em 18/01/10 Aceito para publicação em 25/04/11 Publicado em 20/08/11

Gustavo Aguiar Campana'; Carmen Paz Oplustil'; Lorena Brito de Faro ${ }^{3}$

\section{unitermos resumo}

Medicina laboratorial

A patologia clínica/medicina laboratorial é uma especialidade direcionada à realização de exames complementares no auxílio ao diagnóstico, com impacto nos diferentes estágios da cadeia de saúde:

Medicina diagnóstica prevenção, diagnóstico, prognóstico e acompanhamento terapêutico. Diversos elementos apontam

Patologia clínica para maior utilização da medicina diagnóstica no futuro. Para discutirmos as principais tendências na medicina laboratorial, descrevemos os fatores que colaboram e são fundamentais para o crescimento desse mercado denominados, neste estudo, drivers de crescimento. As principais tendências que terão forte impacto na medicina laboratorial, e que serão descritas neste artigo, são: ferramentas de gestão, inserção de novos testes no mercado e rol de procedimentos, qualidade dos serviços em medicina diagnóstica, modelos de operação, automação, consolidação e integração, tecnologia da informação, medicina personalizada e genética. Sabemos que a medicina diagnóstica demonstra sua importância ao participar de $70 \%$ das decisões clínicas, absorvendo uma pequena parte dos custos em saúde (cerca de $10 \%)$. Todas as tendências analisadas neste trabalho apontam para um crescimento na utilização dos exames laboratoriais e também para sua importância na cadeia de saúde. Esse novo posicionamento, somado às novas expectativas de alta resolubilidade, pressiona o mercado e as companhias que o compõem a buscar mudanças e novas estratégias de atuação.

abstract

Clinical pathology/laboratory medicine, a specialty focused on performing complementary tests to aid diagnosis, has impact upon several stages of health care: prevention, diagnosis, prognosis, and therapeutic management. There are several factors that will foster the use of laboratory medicine in the future. In order to discuss the main trends in laboratory medicine, this article describes the major factors that have promoted growth in this market, which herein are referred to as growth drivers. The major trends that will cause substantial impact on laboratory medicine are: management tools, inclusion of new tests and procedures, service quality, operational models, automation, consolidation and integration, information technology, personalized and genetic medicine. Laboratory medicine occupies a pivotal role in $70 \%$ of all clinical decisions with minimal healthcare costs of approximately $10 \%$. All trends discussed herein sustain an increase in the use of laboratory tests as well as its importance in health care. Both this new model and the expectation of optimal solutions have led the market to search for changes and new management strategies. key words

Laboratory medicine

Diagnostic medicine

Clinical pathology

Automation

Market

1. Especialista em Patologia Clínica; sócio-diretor da Formato Clínico.

2. Mestra em Microbiologia; biomédica; sócia-diretora da Formato Clínico.

3. Médica patologista clínica. 


\section{Introdução}

A patologia clínica/medicina laboratorial é uma especialidade direcionada à realização de exames complementares no auxílio ao diagnóstico, com impacto nos diferentes estágios da cadeia de saúde: prevenção, diagnóstico, prognóstico e acompanhamento terapêutico. Em conjunto, os laboratórios de patologia clínica/medicina laboratorial e os de anatomia patológica, as clínicas de radiologia e imagem, os centros de diagnóstico e a indústria de diagnóstico formam o mercado da medicina diagnóstica.

Embora haja poucos dados publicados sobre a dinâmica desse mercado, referente aos prestadores de serviços, o Cadastro Nacional de Estabelecimentos de Saúde (CNES) estima que seja composto por cerca de 20 mil instituições e que represente um porte de aproximadamente $R \$ 10$ biIhões anuais. Apesar do início de um processo de consolidação desse mercado, o consideramos bastante pulverizado.

Em dezembro de 2008, o país possuía 40.910.631 beneficiários de planos de saúde (excluindo os planos unicamente odontológicos) e estes utilizaram, em média, 11,15 exames por beneficiário(2). Estima-se, portanto, uma demanda no país pela assistência privada de cerca de 450 milhões de exames ao ano. Além desse volume, os exames da rede pública são a maioria. É importante ressaltarmos que o Sistema Único de Saúde (SUS) financia a maior parte da população, mas não cobre sua assistência, direcionando parte de seus gastos para a iniciativa privada.

Diversos elementos indicam maior utilização da medicina diagnóstica no futuro. Atualmente, ela responde por cerca de $80 \%$ das decisões médicas, absorvendo apenas $11 \%$ dos custos em saúde ${ }^{(8)}$

Para discutirmos as principais tendências na medicina laboratorial, descreveremos os fatores que colaboram e são fundamentais para o crescimento desse mercado, denominados, neste artigo, drivers de crescimento.

Em primeiro lugar, o envelhecimento e, por consequência, uma grande modificação no perfil da população brasileira são os maiores desafios para toda a cadeia de saúde.

No censo de 2007, o Brasil apresentou população de 183.987.291 indivíduos e estima-se que, em 2050, esta será de cerca de 220 milhões de brasileiros. O perfil de distribuição das faixas etárias será a principal mudança observada. Um bom exemplo dessa significativa alteração é a população acima de 70 anos de idade, que em 2008 representava $7,95 \%$ do total e em 2050 será responsável por $32,9 \%$ da população ${ }^{(12)}$.
A evolução prevista da pirâmide etária no Brasil, que demonstra essas variáveis discutidas anteriormente, está demonstrada na Figura 1.

Com o maior envelhecimento da população, prevemos maior prevalência de doenças crônicas, elevando a necessidade de realização de exames por essa população(29). $\mathrm{O}$ impacto do envelhecimento da sociedade ocorre também nos custos da saúde. Segundo o Centro de Controle de Doenças (CDC), o custo de promoção da saúde para um idoso norte-americano é três a cinco vezes o custo de saúde de um adulto com menos de 65 anos de idade ${ }^{(5)}$. A maior utilização dos serviços de saúde pela população idosa projeta um aumento na sinistralidade das operadoras de saúde e, consequentemente, maior pressão por custos sob os prestadores de serviços.

Outro importante fator é a elevação da renda da população e o maior índice de empregos formais, que possibilitam maior acesso à assistência à saúde por parte da população brasileira, sendo também considerados importantes drivers de crescimento desse setor.

$\mathrm{O}$ aumento do número de beneficiários de planos de saúde é proporcionalmente maior que a taxa de crescimento da população. Entre dezembro de 2004 e dezembro de 2008, a população brasileira cresceu 5,9\%, enquanto o número de vidas com planos de saúde apresentou $22,3 \%$ de incremento, chegando a mais de 50 milhões de beneficiários ${ }^{(2)}$, demonstrando a resultante dos drivers citados anteriormente.

No que se refere à renda da população, observamos queda da proporção de pobreza e aumento da classe média brasileira, que passou a ser grande consumidora de produtos e serviços e responsável por uma parte significativa do produto interno bruto (PIB) nacional. Correlacionamos o aumento do PIB per capita no Brasil com a proporção de beneficiários de saúde privada na Figura 2.

Quanto ao número de empregos, 76,7\% dos planos de saúde vendidos são planos coletivos (empresariais), relacionando, assim, a assistência à saúde com a taxa de formalidade.

Há também uma mudança no perfil do paciente, que passou a ser considerado um fator decisivo na utilização da medicina. A facilidade de acesso à informação permite que o usuário do mercado de saúde conheça e entenda mais das atualidades e evoluções na área médica e também de sua condição como paciente, tendo como consequência maior compartilhamento e participação nas decisões terapêuticas ${ }^{(24)}$. 


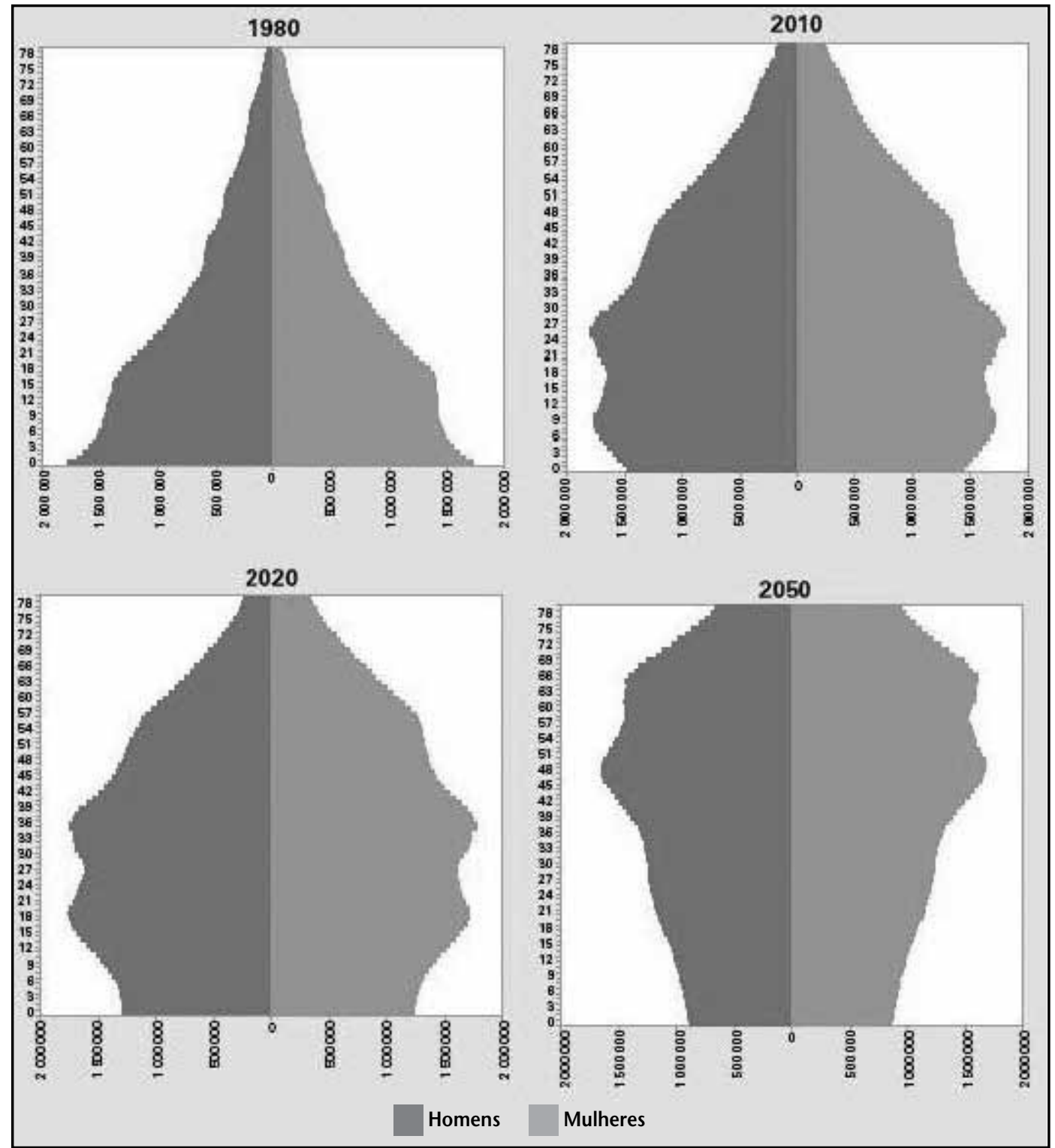

Figura 1 - Composição absoluta da população por idade e sexo (Brasil)

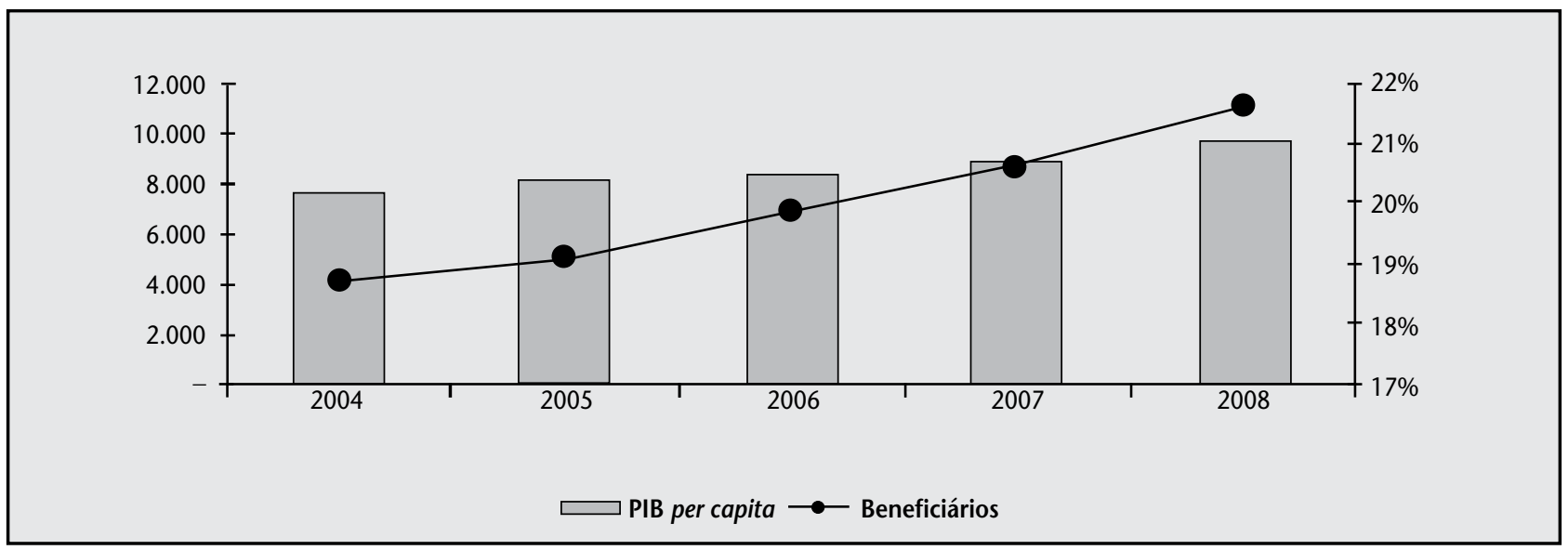

Figura 2 - PIB per capita e beneficiários de OPAS (2004 a 2008)

PIB: produto interno bruto; OPAS: Organização Pan-Americana da Saúde. 
O próprio foco de atuação da assistência à saúde será um fator de modificação do setor. A medicina cada vez mais estará focada na prevenção da doença e na promoção da saúde. As doenças crônicas são responsáveis por $60 \%$ das mortes no mundo, apresentam impacto significativo na qualidade de vida de seus portadores e possuem efeito direto na economia das famílias, comunidades e sociedades ${ }^{(28)}$.

O impacto dessas doenças nas economias ocorre de forma direta, por meio dos gastos com saúde, e de forma indireta, por redução de qualidade e produtividade laborais ${ }^{(28)}$, invalidez e perda de função social e independência( ${ }^{(5)}$. As estimativas de perda de receita proveniente das doenças crônicas, de forma direta e indireta, foram para o Brasil, em 2005, na ordem de US\$2,7 bilhões; em 2015, calcula-se que seja de US\$9,3 bilhões. Estima-se que a perda acumulada até $o$ ano de 2005 foi de US\$ 49,2 bilhões $^{(24)}$. Essa redução representa quase $1 \% \mathrm{em}$ termos de PIB nacional(24).

Na assistência privada à saúde, calcula-se que pacientes portadores de doenças crônicas representam aproximadamente $10 \%$ dos usuários de planos de saúde e são responsáveis por $45 \%$ dos custos das operadoras ${ }^{(26)}$.

Por fim, a utilização de testes laboratoriais direcionados à prevenção de doenças será cada vez maior nos laboratórios, suportada pela medicina baseada em evidências e apoiada na pressão por redução dos custos em saúde(4).

\section{Tendências na medicina laboratorial}

A medicina laboratorial mudou bastante nos últimos anos. O avanço de tecnologias e a grande pressão sobre os custos se traduzem na máxima "fazer um número maior de exames com menos equipamentos, pessoas, tempo, etc.".

Analisaremos, na sequência, as principais tendências que terão forte impacto na medicina laboratorial.

\section{Ferramentas de gestão}

A gestão em medicina diagnóstica por meio de modernas ferramentas administrativas é uma realidade do mercado. O número de publicações citando as mais diversas formas e ferramentas de gestão, modificando o foco das rotinas técnicas para os fluxos de processos das diferentes áreas das empresas, cresceu vertiginosamente.

Destacamos duas das principais ferramentas (ou modelos de negócios) utilizadas em medicina diagnóstica: o Lean Thinking, modelo de gestão com base em mapas de valor e eliminação de desperdícios, difundido pela Toyota e focado em velocidade e eficiência, e o Six Sigma, ferramenta estatística de análise de variações, introduzida pela Motorola, focada principalmente em precisão e acurácia ${ }^{(15)}$.

Diversos artigos publicados demonstram os resultados desses modelos, comprovando seu benefício para as operações técnicas, mediante a diminuição de erros, para os processos, melhorando a oferta dos serviços, e economicamente, gerando melhores margens para as empresas ${ }^{(17)}$.

A utilização dessas ferramentas de gestão extrapola a medicina laboratorial e abrange todo o sistema de saúde, sendo discutida como potencial modelo de melhoria na qualidade em saúde, reduzindo os erros que levam milhares de pacientes a sequelas ou óbitos por tratamentos e diagnósticos não acurados, muitas vezes com base até em problemas regulatórios e de mercado ${ }^{(22)}$.

A ferramenta Six Sigma passou a ser utilizada a partir dos anos 1990 em serviços médicos, iniciando suas implementações em hospitais nos EUA. Sua estratégia é monitorar o processo e mantê-lo sob estabilidade, por meio de classificação, padronização e comparação dos processos, resultando em metodologias de busca da melhoria contínua ${ }^{(3)}$.

Outras ferramentas já foram estudadas e também demonstraram resultados importantes quando aplicadas em empresas de saúde.

\section{Inserção de novos testes no mercado e rol de procedimentos}

Devido ao crescimento das pesquisas na área médica, com base cada vez mais na genética, o uso dos testes laboratoriais será mais frequente e abrangerá todas as fases da cadeia de saúde: prevenção, diagnóstico, prognóstico e acompanhamento terapêutico.

Após a ll Guerra Mundial, com o advento de imunoensaios, automação, computação e técnicas moleculares, o número de testes laboratoriais nos EUA cresceu em $12 \%$ ao ano ${ }^{(4)}$.

A evolução de testes e metodologias laboratoriais sofre diferentes influências já reportadas, como regulamentações, remuneração, mudanças na prática médica, tecnologia diagnóstica, custos e práticas de mercado ${ }^{(13)}$.

A educação orientada aos médicos referente à solicitação dos exames também será cada vez mais abrangente. Nos EUA, estudos demonstraram que apenas dois terços das universidades de medicina lecionavam medicina laboratorial(4). O resultado dessa baixa penetração pode ser traduzido pela significativa quantidade de exames solicitados com impacto pouco significativo no diagnóstico médico ou, ainda, pelos testes obsoletos que constam no rol das agências reguladoras e são comumente solicitados aos laboratórios em geral. 
O aumento dos novos testes disponíveis ocorrerá de forma acelerada. As técnicas moleculares dominarão a medicina laboratorial, serão automatizados, com preços acessivos e orientados à medicina personalizada ${ }^{(4)}$.

A utilização da avaliação de tecnologias em saúde (ATS) permitirá a padronização e a inserção no mercado de testes que apresentem custo-efetividade, definidas por meio de estudos abrangentes de custos, resultados (segurança e benefícios à saúde) e impactos (ético, social e organizacional)(1). Com a ferramenta ATS, haverá também um processo definido e padronizado de inclusão de novas tecnologias no rol de procedimentos da Agência Nacional de Saúde Suplementar (ANS), órgão de regulamentação da saúde suplementar no Brasil. A Figura 3 demonstra o fluxo de inclusão de novas tecnologias no mercado(1).

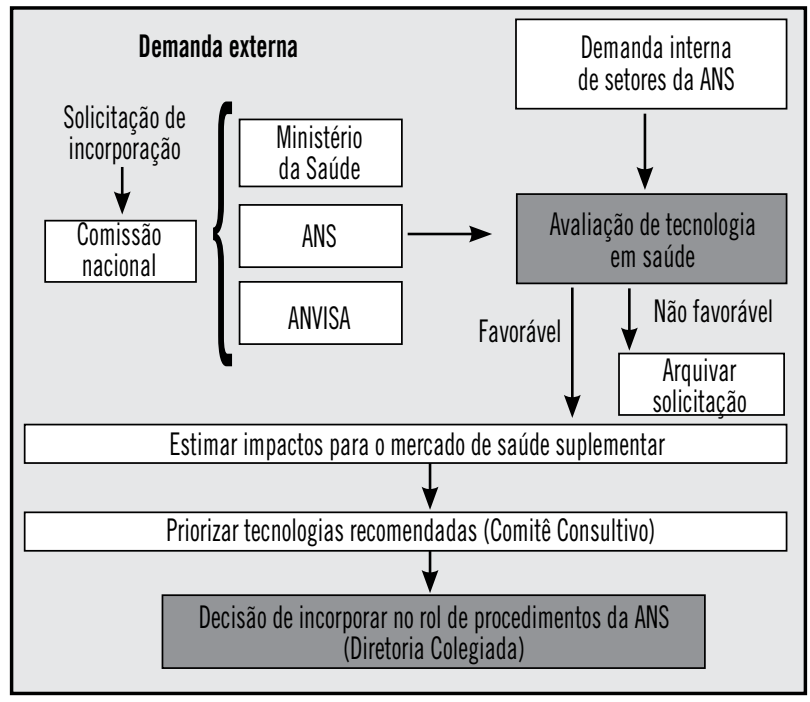

Figura 3 - Fluxo de inclusão de novas tecnologias (ANS)

ANS: Agência Nacional de Saúde Suplementar; ANVISA: Agência Nacional de Vigilância Sanitária.

A ANS definiu uma nova versão em 2008 dos procedimentos que obrigatoriamente devem ser cobertos para todos os beneficiários. Nesse rol, estão cerca de 2.900 itens. Essa ação é pautada na cobertura e não na remuneração e, na visão das operadoras, aumentará os custos assistenciais em até $10 \%$, fato não endossado pela $\mathrm{ANS}^{(26)}$. Essa percepção de aumento na sinistralidade tende a intensificar a pressão por custos sobre os prestadores.

A velocidade da descoberta de novas tecnologias médicas aumenta a cada ano. Nos EUA, um estudo realizado com número significativo de laboratórios, em 1998, demonstrou que 55\% destes haviam descontinuado algum tipo de exame nos últimos dois anos, sendo $75 \%$ dos casos devido a mudanças de tecnologia, seguidos por fatores de regulamentação. Citou-se, ainda, que $39 \%$ das empresas haviam incluído pelo menos algum teste no menu praticado. As razões do acréscimo desses exames foram em $22 \%$ dos casos para melhorar o gerenciamento dos pacientes, $19 \%$ para atender as necessidades dos clientes e comunidades atendidas, $13 \%$ devido a melhores tecnologias disponíveis no mercado e $11 \%$ pelo teste ser mais apropriado para determinado diagnóstico com base em novos conhecimentos médicos. O custo e a margem por exame foram citados em $24 \%$ dos casos como causa secundária de decisão(13).

A medicina com base em evidências é uma das principais ferramentas para direcionamento dos testes a serem utilizados para determinados diagnósticos, tendo como base a efetividade dos exames. As principais finalidades das diretrizes são disseminar as melhores práticas, reduzir a variabilidade, elevar a confiabilidade dos testes para a decisão médica, aumentar a qualidade da assistência em saúde e a facilidade de disseminação e desenvolvimento profissional, auxiliar pesquisas clínicas e, por fim, melhorar a relação custo-efetividade do diagnóstico( ${ }^{(18)}$. No desenvolvimento de diretrizes diagnósticas, não apenas devem ser levadas em consideração as publicações científicas de alto padrão, mas também informações sociais, éticas, financeiras e considerações regionais ${ }^{(18)}$. Entendemos que esse tópico de novos procedimentos e testes obsoletos merece um artigo de revisão específico.

\section{A qualidade dos serviços em medicina diagnóstica}

A qualidade em assistência à saúde é percebida como fator qualificador de posicionamento e permanência no mercado. Hoje, há forte preocupação dos laboratórios em buscar formas de acreditação ou certificação da qualidade, ação apoiada e promovida pelas sociedades da classe.

Em 1999, a Associação Mundial de Sociedades de Patologia e Medica Laboratorial (WASPALM) e a Federação Internacional de Química Clínica (IFCC) publicaram uma declaração sobre a acreditação de laboratórios clínicos referindo que "é do interesse dos pacientes, da Sociedade e do Governo que os laboratórios clínicos operem dentro de altos padrões de competência profissional e técnica devida"(27). A saber:

- as decisões quanto a diagnóstico, prognóstico e terapêutica têm, frequentemente, base nos resultados ou na interpretação de exames laboratoriais e, portanto, danos irreversíveis podem ser causados por resultados errôneos;

- os usuários de serviços de laboratórios, tanto pacientes quanto médicos, podem não possuir conhecimentos técnicos suficientes para avaliar se 
um laboratório está operando em nível satisfatório de qualidade;

- os pacientes e, em menor grau, os médicos podem não ter opção quanto a qual laboratório utilizar;

- os exames de laboratório podem ser dispendiosos e pacientes, seguradoras ou governo, que pagam os exames, têm o direito de esperar que o laboratório forneça informações válidas;

- é do interesse dos laboratórios que sua competência seja atestada por processo de auditoria, por comparação com padrões apropriados e que isso se torne público.

Essa mesma declaração afirma que "a acreditação é uma auditoria externa da capacidade do laboratório em oferecer serviços de alta qualidade"(9), relacionando, assim, a qualidade dos serviços ofertados com a segurança do paciente.

Qualidade, nesse contexto, pode ser definida como um conjunto de características de uma entidade, que se relacionam com sua habilidade em satisfazer necessidades implícitas ou estabelecidas. É fornecer ao cliente informação clinicamente efetiva de maneira eficaz, sob o ponto de vista custo-benefício ${ }^{(20)}$.

Diferentes modelos de programas de acreditação e certificação estão disponíveis no mercado e devem ser direcionadores dos processos das empresas de medicina diagnóstica. Como exemplo, podemos citar órgãos nacionais como o Programa de Acreditação de Laboratórios Clínicos (PALC) ${ }^{(21)}$ da Sociedade Brasileira de Patologia Clínica/Medicina Laboratorial (SBPC/ML) e a Organização Nacional de Acreditação (ONA) ${ }^{(19)}$ e internacionais como os programas do College of American Pathologists $(\mathrm{CAP})^{(6)} \mathrm{e}$ o Joint Comission $(\mathrm{JCl})^{(25)}$.

\section{Modelos de operação: consolidação, associações, entre outros}

Uma das principais discussões atuais refere-se aos novos movimentos do mercado de medicina diagnóstica. A pressão por custos e a dificuldade de acompanhamento nos investimentos em tecnologia realizados pelos líderes de mercado corroboraram para que as empresas familiares, maioria entre as empresas de medicina diagnóstica, iniciassem diferentes modelos de operação para sustentar-se ou garantir sua permanência no mercado. Observamos a partir daí uma série de novas ações realizadas, como abertura de capital para o mercado, associação em clusters de produção, aquisições de empresas por prestadores (consolidação) e operadoras de saúde (verticalização), terceirização das áreas técnicas etc. ${ }^{(9)}$.
Esse mesmo movimento ocorreu por todo o mundo, com instituições crescendo de forma expressiva tanto organicamente como por aquisições, como Labcorp Inc. nos EUA, BML Diagnostics no Japão e Unilabs/Capio atuante em praticamente toda a Europa, e também formando empresas que extrapolam as dimensões continentais, como Quest Diagnostics Inc., presente nos EUA e na Índia e Sonic Healthcare, atuante nos países da Oceania e recentemente nos EUA.

Podemos considerar o mercado de medicina diagnóstica no Brasil bastante pulverizado, com, segundo o CNES, cerca de 20 mil empresas atuantes, apesar de a maioria do volume de exames disponível para o setor privado ser absorvida por número reduzido de empresas. Estimamos que o crescimento dos laboratórios de análises clínicas, em média, esteja em torno de $10 \%$ ao ano, com base no crescimento de vendas da indústria de diagnóstico in vitro, segundo dados da Câmara Brasileira de Diagnóstico (CBDL).

$\mathrm{O}$ aumento das classes econômicas $\mathrm{C}$ e $\mathrm{D}$ levou as empresas a focar investimentos e atuações nesse ramo, oferecendo exames a preços acessíveis e abrangendo a população não beneficiária de assistência privada e que encontra dificuldades no atendimento público.

O processo de consolidação do mercado de medicina diagnóstica, ainda que incipiente, deverá continuar pelas próximas décadas e possivelmente atrairá novos grupos interessados nesse modelo de crescimento. Entendemos como principal benefício da consolidação a velocidade de crescimento e de geração de escala para as companhias alcançarem seus objetivos de custos e sinergias.

A associação entre diversas empresas nos denominados clusters de produção traz como principais benefícios a redução de custos diretos e a troca de experiências técnicas e de gestão; e deve continuar como uma das tendências desse mercado e ampliar seu escopo para maior integração de diferentes áreas das empresas, alcançando sinergias em setores administrativos, por exemplo. Esse modelo deve ter sua base de crescimento e de atuação de forma regional(15).

Outra tendência é o aumento das empresas denominadas nicho, isto é, aquelas que se posicionam de forma específica em seus produtos e serviços, oferecendo testes diferenciados e tecnologias novas.

\section{Automação, consolidação e integração}

Tradicionalmente, os laboratórios operam de forma departamentalizada e orientada por serviços (bioquímica, endocrinologia, hematologia etc.). Esse modelo de 
operação demonstrou ser pouco eficiente e, por outro lado, a consolidação e a integração entre as áreas geram redução dos custos dos laboratórios e tende a aumentar com a utilização da automação pré-analítica ${ }^{(4)}$.

Automação laboratorial consiste na integração entre hardware e software desenhados para o processamento e a análise por completo das amostras ${ }^{(7)}$ e evoluiu de uma operação com base em aspectos mecânicos nos anos 1970 para um sistema orientado por informações complexas na década de $1990^{(14)}$.

Os métodos automáticos foram introduzidos na patologia clínica/medicina laboratorial nos anos 1950 pela Technicon por meio dos conceitos de fluxo contínuo ${ }^{(16)}$. Uma das principais evoluções foi a utilização de equipamento multicanal, que permitiu a realização de dosagens de diversas substâncias em únicas plataformas ${ }^{(16)}$.

Atualmente, a maioria do volume processado nos laboratórios de análises clínicas está contemplada pelos equipamentos automatizados. Apesar de estimarmos mais de 5 mil tipos diferentes de exames disponíveis no mercado, um número reduzido destes é responsável por mais de $80 \%$ das rotinas técnicas em laboratórios com perfis ambulatoriais e hospitalares. As empresas voltadas para o mercado de apoio ou referência laboratorial apresentam um mix de exames diferente, sendo que as técnicas manuais ou "especializadas", como radioimunoensaio, biologia molecular, cromatografia, entre outras, absorvem uma parte significativa do total de exames processados. A consolidação de um número maior de tipos de ensaio e metodologia em equipamentos gera resultados importantes aos laboratórios em termos de produtividade, diluição de custos de insumos acessórios, menor manipulação de amostras, menor número de tubos por pacientes e, consequentemente, menor chance de erros.

A automação em análises clínicas pode ser dividida em três principais modelos de operação, com base na disposição dos hardwares envolvidos: Automação Modular (MA), Automação Total (TLA) e Automação Direcionada por Tarefas (TTA). Todas essas modalidades integram, de diferentes formas, as fases pré-analítica, analítica e pós-analítica. A implementação de um tipo ou outro de automação requer estudo detalhado e avaliação de variáveis diversas, como volume de exames, mix de exames, atributos estratégicos desejados para a operação, níveis de serviços acordados com clientes, capacidade instalada e previsão de crescimento, necessidade de investimento, retorno sobre o capital investido e custo-benefício.

A escolha do modelo de operação deve considerar também o processo de produção do laboratório, sua relação com a assistência em saúde e o foco da empresa como negócio ${ }^{(14)}$, resultando em indicadores financeiros (preferencialmente retorno sobre investimento [ROI]), operacionais e de melhoria dos processos ${ }^{(30)}$. A descrição de cada uma das modalidades de automação merece um artigo específico, assim como os benefícios da automação nos laboratórios de patologia clínica. Os principais pontoschave para a implementação de automação laboratorial são: envolvimento de todas as partes relacionadas (stakeholders), com destaque para os funcionários e a equipe, justificativas financeiras e de investimentos, utilização de ferramentas de gerenciamento de projetos, alinhamento com o sistema de informação laboratorial (LIS), definição de mix de exames a ser processado, estudo de logística de materiais, entre outros $^{(30)}$.

Ressaltamos que a evolução da automação laboratorial, em todas as suas fases, está intimamente relacionada com a evolução em tecnologia da informação (TI), incluindo os processos automáticos pós-analíticos, por meio de fluxogramas de interpretação (delta check, lógica fuzzy etc.), protocolos de repetição e diluição de exames, testes reflexos etc. ${ }^{(14)}$.

\section{Tecnologia da informação}

A TI é uma das principais forças de mudança no mercado de medicina diagnóstica e permitirá uma integração cada vez maior dos diferentes players e segmentos da cadeia, como fontes pagadoras, prestadores, fornecedores etc. ${ }^{(9)}$, e um controle mais eficaz da operação em todos os seus níveis, auxiliando na geração de valor a todas as partes relacionadas.

A seguir, são apresentadas as principais tendências e modificações estruturais que a TI trará para o mercado de medicina diagnóstica. Direcionamento da informação do laboratório clínico do futuro ${ }^{(9)}$ :

- integração técnica e organizacional dos processos laboratoriais e gerenciamento da informação;

- evolução dos laboratórios para organizações virtuais/digitais desenhadas para relacionar-se com redes descentralizadas;

- evolução das bases de LIS para arquiteturas com base na internet;

- evolução dos laudos com base em texto para bases de imagens e internet;

- ênfase na necessidade de informação dos clientes (médicos e pacientes);

- maior preocupação com segurança, confidencialidade e legislações. 
Um importante exemplo de evolução da Tl em saúde é o estabelecimento, por parte da ANS, de um padrão de Troca de Informação em Saúde Suplementar (TISS), para registro e intercâmbio de dados entre as operadoras e os prestadores de saúde, prevendo benefícios, como maior velocidade na comunicação, redução do uso de papel, facilidade na obtenção de dados para estudos epidemiológicos, realização de análises de custos, benefícios e investimentos, redução de custos administrativos, comparação de desempenho entre instituições e melhoria na qualidade da assistência à saúde. Destacamos, aqui, os objetivos da TISS e não os resultados alcançados até o momento(2).

\section{Medicina personalizada e genética}

A medicina personalizada também é uma das tendências da assistência médica no mundo. Podemos defini-la como a utilização da genética e das características individuais como direcionadora dos métodos diagnósticos e terapêuticos. Os principais exemplos da medicina personalizada referem-se aos testes genéticos que designam escolhas terapêuticas individuais ${ }^{(10)}$. Os testes genéticos já validados devem ser submetidos a estudos de sensibilidade, especificidade e valores preditivos em relação aos genótipos (validação analítica) e resultados assistenciais (validação clínica) ${ }^{(23)}$, assim como deve ser definido o custo-benefício de cada um desses ensaios, direcionando, principalmente para a população-alvo, implicações sociais, fenotípicas e de resultados financeiros ${ }^{(11)}$.

Algumas dificuldades e barreiras existem para a introdução dessa medicina na prática, como descobertas e definições que doenças de alta prevalência, como neoplasias, doenças cardíacas e diabetes, têm como base etiológica os componentes genéticos complexos e não mendelianos, dificultando a identificação dessas mutações. Variantes de múltiplos genes contribuem para uma pequena parte do risco total do paciente ${ }^{(23)}$.

Diversas dificuldades são encontradas na introdução desses testes nas rotinas médicas, como o relacionamento com as fontes pagadoras, pois ainda são testes de alto valor, e a necessidade de estudos clínicos que demonstrem claramente o benefício da medicina personalizada ${ }^{(10)}$.

A evolução dos testes moleculares tornará essa metodologia acessível aos laboratórios clínicos, por meio de screenings de predisposições genéticas às doenças específicas, detecção de doenças infecciosas e determinação de expressões gênicas e moleculares. Prevemos também a integração dessas metodologias à automação já existente, introduzindo esses ensaios nas rotinas laboratoriais( ${ }^{(4)}$.
$\mathrm{O}$ advento desses testes genéticos implica em uma compreensão de qualidade de vida pelos pacientes de forma ambígua, podendo ser positiva pela possibilidade de prevenir a doença ou negativa pelo conhecimento da probabilidade de desenvolvê-la. Além disso, devem ser considerados estudos de avaliação de tecnologias em saúde ${ }^{(11)}$.

\section{Discussão}

As tendências de crescimento da medicina laboratorial fazem parte de um grupo importante de mudanças que a própria evolução da medicina alcançou. Os desafios para lidar com esse novo público, melhorando a qualidade da assistência à saúde e tornando-a realmente eficaz, certamente é um dos principais desafios daqui para frente.

A pressão por mudanças práticas e conceituais acontece em todas as pontas de relacionamento desse setor, como o cliente que passa a ser mais exigente quanto ao nível de produtos e serviços; os fornecedores e as fontes pagadoras por meio da pressão por custos e preços, respectivamente; a comunidade médica depositando cada vez mais confiança nos testes laboratoriais; os acionistas exigindo maior retorno sobre seu capital; e toda a dinâmica de mercado forçando as empresas a se diferenciar de seus concorrentes e oferecer melhores soluções a seus clientes.

As recentes mudanças na economia global e a rápida evolução da área médica, em especial no que se refere ao setor de diagnóstico, permitem que façamos observações quanto às tendências do mercado, dos modelos de operação e da tecnologia empregada. Uma das principais tendências da medicina laboratorial é a atuação de seus profissionais como consultores em saúde, apoiando à prática clínica na assistência à saúde, com base no avanço da tecnologia e na complexidade médica.

Demonstramos, a seguir, as tendências (previsões para o século XXI) para a medicina laboratorial discutidas na literatura médica ${ }^{(4)}$.

- Ambiente do mercado de saúde:

$\sqrt{ }$ reforma com base na fonte pagadora (unificação);

$\sqrt{ }$ avanços contínuos em ciência e tecnologia;

$\sqrt{ }$ redes regionais de assistência à saúde integradas;

$\sqrt{ }$ maior ênfase nos testes remotos (POIC);

$\checkmark$ telemedicina e práticas médicas on-line;

$\sqrt{ }$ maior ênfase na contenção de custos. 
- Prática médica:

$\sqrt{ }$ maior ênfase na medicina preventiva;

$\sqrt{ }$ atenção primária à saúde na prática de enfermagem;

$\sqrt{ }$ gerenciamento de doenças com base em evidências.

- Tecnologia laboratorial:

$\sqrt{ }$ automação das tecnologias moleculares;

$\sqrt{ }$ plataformas de operação integradas;

$\sqrt{ }$ automação de laboratórios regionais;

$\sqrt{ }$ automação modular.

- Prática da medicina laboratorial:

$\sqrt{ }$ laboratório abrangendo serviços-consultores;

$\sqrt{ }$ gerenciamento da Tl; $\sqrt{ }$ programas de gerenciamento de doenças com base em evidências;

$\sqrt{ }$ escolha dos laboratórios pelos pacientes;

$\sqrt{ }$ maior ênfase na subespecialização;

$\sqrt{ }$ integração das especialidades diagnósticas.

Sabemos que a medicina diagnóstica demonstra sua importância por participar em 70\% das decisões clínicas, absorvendo uma pequena parte dos custos em saúde (em torno de 10\%). Todas as tendências analisadas neste artigo apontam um crescimento na utilização dos exames laboratoriais e também de sua importância na cadeia da saúde. Esse novo posicionamento, somado às novas expectativas de alta resolubilidade, pressiona o mercado e as companhias que o compõem a buscar mudanças e novas estratégias de atuação.

\section{Referências}

1. AGÊNCIA NACIONAL DE SAÚDE SUPLEMENTAR (ANS). Caderno de informação da saúde suplementar. Dez./2008. Disponível em: <www.ans.org.br>.

2. AGÊNCIA NACIONAL DE SAÚDE SUPLEMENTAR (ANS). Caderno de informação da saúde suplementar. Mar./2009. Disponível em: <www.ans.org.br>.

3. BERLITZ, F. A.; HAUSSEN, M. L. Seis sigma no laboratório clínico: impacto na gestão de performance analítica dos processos técnicos. JBPML, v. 41, n. 5, p. 301-12, 2005.

4. BURKE, D. Laboratory medicine in the $21^{\text {st }}$ century. Am J Clin Pathol, v. 114, n. 6, p. 841-6, 2000.

5. CENTER OF DISEASE CONTROL (CDC). The state of aging and health in America. 2007. Disponível em: <www. cdc.gov>.

6. COLLEGE OF AMERICAN PATHOLOGISTS (CAP). Disponível em: <www.cap.org>.

7. FELDER, R. Automation: survival tools for the hospital laboratory. Second International Bayer Diagnostics Laboratory Testing Symposium, 1998.

8. FORSMAN, W. F. Why is the laboratory an afterthought for managed care organizations? Clin Chem, v. 42, n. 5 , p. 813-6, 1996.

9. FRIEDMAN, B. The total laboratory solution: a new laboratory e-business model based on a vertical laboratory metanetwork. Clin Chem, v. 47, n. 8, p. 1526-35, 2001.

10. GARBER, A. M.; TUNIS, S. R. Does comparative effectiveness research threaten personalized medicine? NEJM, v. 360, n. 19, p. 1925-7, 2009.

11. GOLDIE, S. J.; LEVIN, A. R. Genomics in medicine and public health: role of cost-effectiveness analysis. JAMA, v. 286, n. 13, p. 1637-8, 2001.

12. INSTITUTO BRASILEIRO DE GEOGRAFIA E ESTATÍSTICA (IBGE). Projeção da população do Brasil por sexo e idade: 1980 a 2050. Revisão 2008. Disponível em: $<$ www.ibge.gov.br>.

13. LABEAU, K. M. et al. Clinical laboratory tests menu changes in the pacific northwest: 1994 to 1996. Clin Chem, v. 44, n. 4, p. 833-8, 1998.

14. MARKIN, R. S.; WHALEN, S. A. Laboratory automation: trajectory, technology and tactics. Clin Chem, v. 46, n. 5, p. 764-71, 2000.

15. MICHAEL, R. L.; CHRISTENSEN, S. Ten trends that highlights rapid changes in healthcare \& laboratory medicine. Dark Daily Report, 2008.

16. MITCHELL, F. L. Automation in clinical chemistry: developments and recent trends. Jour Aut Chem, v. 1, n. 1, p. 1-9, 1978.

17. NOVIS, D. A.; KONSTANTAKOS, G. Reducing errors in the practice of pathology and laboratory medicine. Am J Clin Pathol, v. 126, Suppl., p. S30-S35, 2006.

18. OOSTERHUIS, W. P. et al. Evidence-based guidelines in laboratory medicine: principles and methods. Clin Chem, v. 50, n. 5, p. 806-18, 2004.

19. ORGANIZAÇÃO NACIONAL DE ACREDITAÇÃO (ONA). Disponível em: <www.ona.org.br>.

20. SHCOLNIK, W. Sociedade Brasileira de Patologia Clínica/ Medicina Laboratorial: programas de qualidade. Disponível em: <www.sbpc.org.br>.

21. SOCIEDADE Brasileira de Patologia Clínica/Medicina Laboratorial (SBCP/ML). Disponível em: <www.sbpc. org.br>.

22. SPEAR, S. J. Fixing healthcare from the inside, today. Harvard Business Review, 2005.

23. STEINBERG, K. K. et al. The role of genomics in public health and disease prevention-report. JAMA, v. 286, n. 13, p. 1635, 2001. 
24. STONE, J. H. Communication between physicians and patients in the era of e-medicine. NEJM, v. 356, n. 24, p. 2451-3, 2007.

25. THE JOINT COMMISSION (JCl). Disponível em: <www. jointcommission.org>.

26. VALOR ECONÔMICO. Análise Setorial 2009. Saúde Suplementar.

27. WORLD ASSOCIATION of Societies of Pathology and Laboratory Medicine (WASPALM). Disponível em: $<$ www.waspalm.org $>$.
28. WORLD HEALTH ORGANIZATION (WHO). Preventing chronic diseases: a vital investment. 2005. Disponível em: <www.who.int>.

29. WORLD HEALTH ORGANIZATION (WHO). The world health report 2008. Primary health care. Now more than ever. 2008. Disponível em: <www.who.int>.

30. YOUNG, D. Laboratory automation: smart strategies and practical applications. Clin Chem, v. 46, n. 5, p. 740-5, 2000. 